\title{
Analytical Modelling of Back-off Process of IEEE 802.11p using Continuous Markov Chain in VANETs
}

\author{
Priyanka, Rishi Pal Singh \\ Dept. of Computer Science \& Engg, \\ Guru Jambheshwar University of Science \& \\ Technology, \\ Hisar (Haryana), India.
}

\author{
Sushil Kumar \\ School of Computer \& Systems Sciences, \\ Jawaharlal Nehru University, \\ New Delhi, India.
}

\begin{abstract}
Recently, the IEEE has standardised the $802.11 \mathrm{p}$ protocol for Vehicular Ad hoc Network (VANET). The main medium access control mechanism (MAC) of IEEE 802.11p is known as Enhanced Distributed Channel Access (EDCA). EDCA uses a contention algorithm based on distributed coordination function to provide the access for traffic in each access category (AC). When the contention window reaches its maximum size, the contention window is reset to its minimum size when frames are transmitted successfully, or the associated retry counter is reached, and the frame is discarded. In this paper, propose an analytical model of the throughput for the IEEE $802.11 \mathrm{p}$ protocol is proposed using continuous time Markov chain (CTMC) with upper bound of drop limit. During transmission, some messages require RTS/CTS prior to transmitting data and others don't require it. Therefore, this idea along with the concept of drop limit is considered for modeling. A 3-D Markov chain is created to model the back off procedure for each access category. The analytical evaluation of the throughput for each access category of IEEE $802.11 \mathrm{p}$ EDCA has been given. The model is applicable for four access categories and the solution is given without including the computational complexity.
\end{abstract}

\section{Keywords}

Vehicular Networks, Throughput, WAVE, IEEE 802.11e EDCA, IEEE 802.11 , retry limits.

\section{INTRODUCTION}

The Vehicular Ad-hoc network was initially designed to provide safety to the car drivers, as the number of road accidents is increasing day by day. The traffic congestion on road is also a big problem which leads to the wastage of fuel and time. The VANET permits each vehicle to efficiently communicate with the nearby or the distant vehicles on the same road or even on the different roads or with the road side units. VANET has significant potential to enable diverse applications associated with traffic safety, traffic efficiency and infotainment. Its unique characteristics include high mobility nodes, rapidly changing network topology, potentially unbound network size, anonymous addressee, and delay sensitivity. The mobility of the nodes depends on the topology and layout of roads, which helps in the prediction of node position and also helps in maintaining the privacy of the nodes. The VANET could include the vehicles in one city, several cities or even a country; therefore, the network topology should be scalable. It is a time sensitive network because data delivery after happening of the accident is not worth [1-2].
The Medium Access Control plays a major role in the performance of the vehicular ad-hoc network. For this purpose, the standard developed by IEEE is IEEE $802.11 \mathrm{p}$, which is an extension of IEEE 802.11e and provides Wireless Access in Vehicular Environments (WAVE). It uses the frequency band of $5.9 \mathrm{GHz}$ with channel spacing of $5 \mathrm{MHz}$, $10 \mathrm{MHz}$, and $20 \mathrm{MHz}$. The IEEE 802.11p is based on ASTM standard E2213-03 and uses ITS band known as DSRC (Dedicated Short Range Communications) [3-5]. The IEEE $802.11 \mathrm{p}$ group was formed in November 2004 and was approved on July 15, 2010, as "Amendment 6: Wireless Access in Vehicular Environments (WAVE)". The WAVE defines the architecture, communication model, management structure, security and physical access. The architectural components of WAVE include On Board Unit (OBU), Road Side Unit (RSU), and WAVE interface [6].

The major concern of MAC layer is the back-off procedure which is dependent on the size of the contention window. Another parameter of MAC layer for IEEE $802.11 \mathrm{p}$ is the arbitrary inter- frame space number (AIFSN). The values of the different parameters like minimum contention window size $\left(C W_{\min }\right)$, maximum contention windows size $\left(C W_{\max }\right)$, and AIFSN of the MAC layer for IEEE 802.11p for four ACs: background (AC_BK), best effort (AC_BE), video (AC_VI), and voice $\left(\mathrm{AC}_{-} \mathrm{VO}\right)$ are given in Table-I.

The Arbitrary Inter Frame Space (AIFS) can be calculated for different access categories by the formula:AIFS $[A C]=$ AIFSN $[A C] *$ aslotTime + aSIFSTime.

Analytical modelling of the MAC sublayer for the IEEE 802.11 and its variant has done by the several authors. The detailed survey of the work related to this area is provided in section II. The modeling will be done by using the continuous time Markov chain.

Markov chain can be defined as a Markov process in either discrete or continuous time along with a countable state space irrespective of the nature of time. In this paper, the continuous time Markov chain is being considered. The continuous time Markov chain can be defined by a countable or finite state space, a transition rate matrix having dimensions equal to the dimensions of state space and the probability distribution.

In this paper, an analytical model of IEEE $802.11 \mathrm{p}$ is presented which reveal its performance for each access category. The 3-D Markov chain is created to model the backoff procedure for each Access Category (AC). The size of the contention window (minimum and maximum) and the AIFSN are considered separately for each access category. 
Table I- MAC layer parameters for IEEE 802.11p [15]

\begin{tabular}{|c|c|c|l|c|c|}
\hline $\mathrm{AC}$ & \multicolumn{1}{|c|}{$\mathrm{CW}_{\min }[\mathrm{AC}]$} & $\begin{array}{c}\text { Value } \\
\left(\mathrm{CW}_{\min }\right)\end{array}$ & \multicolumn{1}{c|}{$\mathrm{CW}_{\max }[\mathrm{AC}]$} & $\begin{array}{c}\text { Value } \\
\left(\mathrm{CW}_{\max }\right)\end{array}$ & AIFSN[AC] \\
\hline $\mathrm{AC} \_\mathrm{BK}$ & $\mathrm{CW}_{\min }$ & 31 & $\mathrm{CW}_{\max }$ & 1023 & 9 \\
\hline $\mathrm{AC} \_\mathrm{BE}$ & $\left(\mathrm{CW}_{\min }+1\right) / 2-1$ & 31 & $\mathrm{CW}_{\max }$ & 31 & 6 \\
\hline $\mathrm{AC} \_\mathrm{VI}$ & $\left(\mathrm{CW}_{\min }+1\right) / 4-1$ & 15 & $\mathrm{CW}_{\max }$ & 1023 & 2 \\
\hline AC_VO & $\left(\mathrm{CW}_{\min }+1\right) / 4-1$ & 7 & $\left(\mathrm{CW}_{\max }+1\right) / 2-1$ & 15 \\
\hline
\end{tabular}

The model in this paper considers four access categories namely: AC0, AC1, AC2 and AC3. The model also takes into account the data packets and the control packets separately. The proposed analytical model has been used to analyse throughput of the IEEE $802.11 \mathrm{p}$ with the consideration of drop limit and blocking probability.

The paper is organized into different sections. Section II briefs the related work which has been done so far. Section III presents the proposed analytical model of IEEE $802.11 \mathrm{p}$ and the expressions for transmission probability and throughput have been derived. The throughput analysis is presented in section IV. The analytical results of the proposed model are presented in section V. Section VI concludes the paper.

\section{2. RELATED WORK}

IEEE 802.11p MAC mechanism is based on EDCA as specified in 802.11e [7]. It is an enhancement of IEEE 802.11 DCF mechanism with QoS (Quality of Service). In this technique, the application packets are divided into queues called access categories. These access categories possess priorities according to the application traffic. Many authors evaluated the QoS performance of vehicles in terms of the mean delay and transmission throughput using EDCA in the highly mobile vehicular environment [8-11]. The evaluation is given by three dimensional Markov chain using spatial zone, current back off stage and current back off time. The concept of Markov chain was initially used in analysing the performance of IEEE 802.11 DCF. The proposed analysis applied to both the packet transmission schemes i.e. basic access mechanism, RTS/CTS mechanism and their combination. The packets longer than the given threshold were transmitted according to the RTS/CTS mechanism. The 2-D discrete time Markov Chain was used for modelling the IEEE 802.11 DCF. The throughput had been computed using this analytic work and RTS/CTS mechanism outperformed basic access mechanism. The Markov chain model has also been utilized to analyse mobility at an intersection by using the concept given by Pipes and Nagel [12-15].

Back off algorithms are considered to be the principal component of MAC. The choice of contention window is very important for any back off algorithm. If back off algorithm selects larger contention window $(\mathrm{CW})$ size in order to reduce the collision probability, the delay will be increased that is not suitable for delay sensitive networks. If back off algorithm chooses smaller $\mathrm{CW}$, the probability of collision will be increased which results in a reduction of throughput. Therefore, there is always a tradeoff between CW size and performance. As per the study is given in the paper [16], Enhanced Binary Exponential Back-off (EBEB) was most suitable for wireless ad hoc network. But this back off algorithm also had some limitations like hidden \& exposed terminals and lack of mobility. The authors in [17] tried to give the idea about improved protocol capacity of IEEE 802.11 network by tuning its back off algorithm and back off window size that balance collision and idle period costs. Geometric distribution was used by the author to give analytical study. From geometric back off assumption, all the processes which define the channel occupancy pattern (i.e. empty slot, collisions and successful transmissions) are regenerative with respect to the sequence of time instants corresponding to the completion of the successful transmission.

The continuous time Markov chain was used for modelling [18-21] in several papers. For this model, the special case of continuous time Markov chain, called birth-death process is used. In this model, there are two types of state transitions known as "births" and "deaths". The state transition "birth" increases the value of state variable by one and the "death" state transition decreases the value of state variable by one. The process is described by using the birth rate and the death rate.

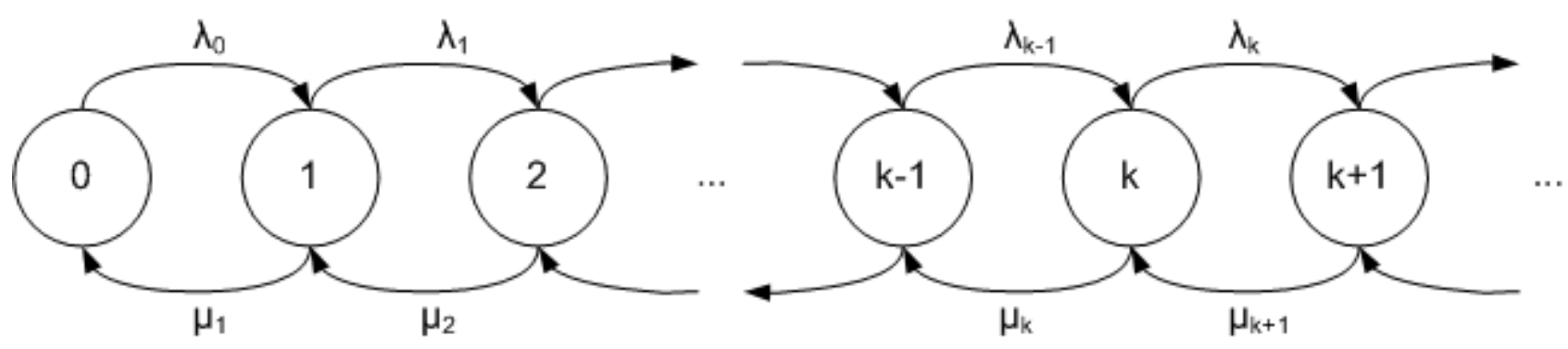

Figure 1: Birth death process 
The performance of IEEE $802.11 \mathrm{p}$ is described by extensive simulations in section V. The symbols used in this paper are listed in Table-II.

Table II: Symbol Notation

\begin{tabular}{|c|l|}
\hline Symbol & Explanation \\
\hline$A C$ & Access Category \\
\hline$A C_{-} B K$ & Access Category__Background \\
\hline$A C_{-} B E$ & Access Category_ Best effort \\
\hline$A C_{-} V I$ & Access Category_ Video \\
\hline$A C \_V O$ & Access Category_ Voice \\
\hline$A I F S$ & Arbitration Inter Frame Space \\
\hline$A I F S N$ & $\begin{array}{l}\text { Arbitration Inter Frame Space } \\
\text { Number }\end{array}$ \\
\hline$E[D]$ & $\begin{array}{l}\text { Average amount of data payload } \\
\text { successfully transmitted in a slot } \\
\text { time }\end{array}$ \\
\hline$j$ & Current number of back-off stage \\
\hline$k$ & Current back-off counter \\
\hline$\tau$ & Transmission probability \\
\hline$\sigma$ & Slot time \\
\hline$\delta$ & Propagation delay \\
\hline &
\end{tabular}

\section{PROPOSED WORK}

Most of the models were used for only two access categories (AC1 and $\mathrm{AC} 3$ ) in order to reduce computational complexity. Our proposed model considers four access categories (AC0, $\mathrm{AC} 1, \mathrm{AC} 2$, and $\mathrm{AC} 3$ ) throughout the analytical modelling which supports all the ACs in IEEE 802.11p.

The stochastic behaviour of the IEEE $802.11 \mathrm{p}$ back-off procedure is represented using the Continuous time Markov chain as shown in Fig. 2. The Saturated traffic conditions are considered in order to reduce the computation complexity and to obtain the accuracy of the model. Following are the assumptions of the proposed model:

1. The network consists of finite contending stations say $n$ and all stations can listen to each other.

2. The Packet collision probability is constant and independent to the retransmission attempts.

3. Each node always has a packet to transmit after every successful transmission.

A node attempts to transmit the control packet (frame) whenever the backoff counter is zero. The packets may collide if more than one node starts transmitting at the same time. It is assumed that the collision probabilities for the different back-off stages are same and are represented by $p$. If there is no collision, it is considered that the packet is successfully transmitted with the probability $\mathrm{p}_{\mathrm{s}}$. The detailed back-off procedure for this model is represented in the figure2.

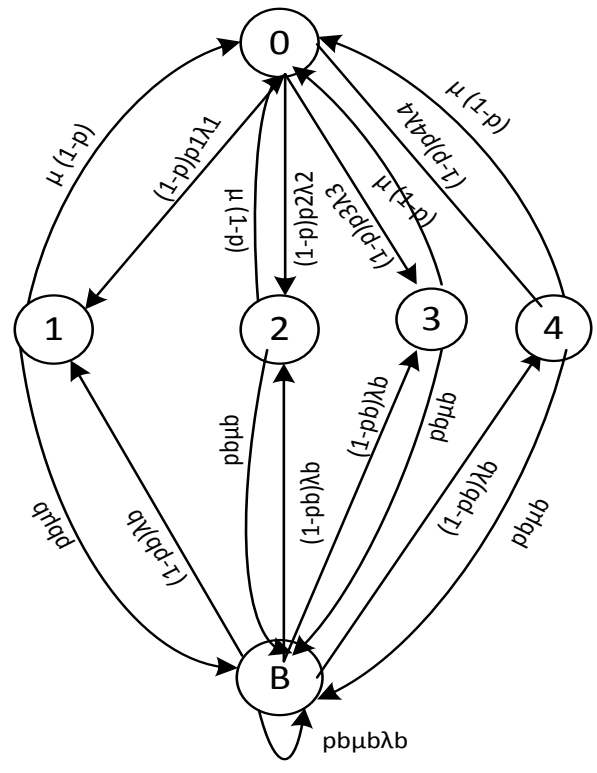

Figure 2: Backoff process

The model consists of 5 states including an idle state, four states representing each access category and the blocking state. The description of each state is given in table III.

TABLE III. Description of States

\begin{tabular}{|c|c|}
\hline State & Description \\
\hline 0 & Idle state \\
\hline 1 & Access Category0-AC_BK \\
\hline 2 & Access Category1-AC_BE \\
\hline 3 & Access Category2-AC_VI \\
\hline 4 & Access Category3-AC_VO \\
\hline$B$ & $\begin{array}{c}\text { The state } B \text { describes the state of } \\
\text { blocking in the network. The transition } \\
\text { probability from the state } B \text { to state } 1,2,3 \\
\text { are shown in the figure } 1 .\end{array}$ \\
\hline
\end{tabular}

The one-step transition probability is defined as the probability of transition from one state to another in a single step. The one step probability matrix helps to easily visualize the transition from one state to another and is used to calculate the steady state probabilities. Here, 0 shows that there is no link between the two states in one step. The onestep transition matrix is given as below using the continuous time Markov chain.

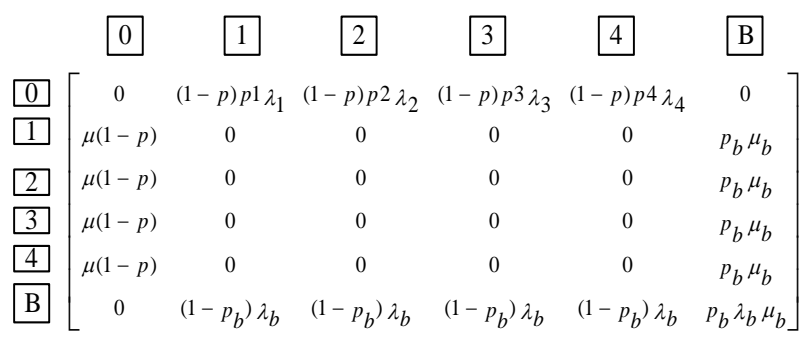

The steady state probability can be defined that the distribution of the system will remain same at every point of time. The Markov process used here is the birth-death process. The expressions using this method are given by equating sum of outgoing links and the sum of the incoming 
links. Equation number 1 to 6 shows the outgoing links from a node on the left-hand side and the incoming links to a node at the right side of the equation. The steady-state probabilities for all the states are given as below

$\pi_{0}(1-p)\left[\left(p 1 * \lambda_{1}\right)+\left(p 2 * \lambda_{2}\right)+\left(p 3 * \lambda_{3}\right)+\left(p 4 * \lambda_{4}\right)\right]=$ $\mu(1-p)\left(\pi_{1}+\pi_{2}+\pi_{3}+\pi_{4}\right)$

$\pi_{1}\left[\mu(1-p)+p_{b} \mu_{b}\right]=\pi_{0}(1-p)\left(p 1 * \lambda_{1}\right)+\pi_{b}\left(1-p_{b}\right) \lambda_{b}$

$\pi_{2}\left[\mu(1-p)+p_{b} \mu_{b}\right]=\pi_{0}(1-p)\left(p 2 * \lambda_{2}\right)+\pi_{b}\left(1-p_{b}\right) \lambda_{b}$

$\pi_{3}\left[\mu(1-p)+p_{b} \mu_{b}\right]=\pi_{0}(1-p)\left(p 3 * \lambda_{3}\right)+\pi_{b}\left(1-p_{b}\right) \lambda_{b}$

$\pi_{4}\left[\mu(1-p)+p_{b} \mu_{b}\right]=\pi_{0}(1-p)\left(p 4 * \lambda_{4}\right)+\pi_{b}\left(1-p_{b}\right) \lambda_{b}$

$\pi_{b}\left[4\left(1-p_{b}\right) \lambda_{b}\right]=p_{b} \mu_{b}\left(\pi_{1}+\pi_{2}+\pi_{3}+\pi_{4}\right)$

As per the normalization condition,

$\pi_{0}+\pi_{1}+\pi_{2}+\pi_{3}+\pi_{4}+\pi_{b}=1$

The final expressions of the steady state probabilities are calculated using equation 1 to 6 and the results are given below.

$\pi_{b}=\frac{p_{b} \mu_{b}\left[\left(p 1 * \lambda_{1}\right)+\left(p 2 * \lambda_{2}\right)+\left(p 3 * \lambda_{3}\right)+\left(p 4 * \lambda_{4}\right)\right]}{4 \mu\left(1-p_{b}\right) \lambda_{b}} \pi_{0}$

$\pi_{1}=$

$\frac{\pi_{0}}{\mu(1-p)+p_{b} \mu_{b}}\left\{(1-p)\left(p 1 * \lambda_{1}\right)+\right.$

$\left.\frac{p_{b} \mu_{b}\left[\left(p 1 * \lambda_{1}\right)+\left(p 2 * \lambda_{2}\right)+\left(p 3 * \lambda_{3}\right)+\left(p 4 * \lambda_{4}\right)\right]}{4 \mu}\right\}$

$\pi_{2}=$

$\frac{\pi_{0}}{\mu(1-p)+p_{b} \mu_{b}}\left\{(1-p)\left(p 2 * \lambda_{2}\right)+\right.$

$\left.\frac{p_{b} \mu_{b}\left[\left(p 1 * \lambda_{1}\right)+\left(p 2 * \lambda_{2}\right)+\left(p 3 * \lambda_{3}\right)+\left(p 4 * \lambda_{4}\right)\right]}{4 \mu}\right\}$

$\pi_{3}=$

$\frac{\pi_{0}}{\mu(1-p)+p_{b} \mu_{b}}\left\{(1-p)\left(p 3 * \lambda_{3}\right)+\right.$

$\left.\frac{p_{b} \mu_{b}\left[\left(p 1 * \lambda_{1}\right)+\left(p 2 * \lambda_{2}\right)+\left(p 3 * \lambda_{3}\right)+\left(p 4 * \lambda_{4}\right)\right]}{4 \mu}\right\}$

$\pi_{4}=$

$\frac{\pi_{0}}{\mu(1-p)+p_{b} \mu_{b}}\left\{(1-p)\left(p 4 * \lambda_{4}\right)+\right.$

$\left.\frac{p_{b} \mu_{b}\left[\left(p 1 * \lambda_{1}\right)+\left(p 2 * \lambda_{2}\right)+\left(p 3 * \lambda_{3}\right)+\left(p 4 * \lambda_{4}\right)\right]}{4 \mu}\right\}$

$\pi_{0}=\frac{4 \mu^{2}\left(1-p_{b}\right) \lambda_{b}}{l}$

Where,

$$
\begin{aligned}
l=4 \mu^{2}\left(1-p_{b}\right) \lambda_{b} & +\left[\left(p 1 * \lambda_{1}\right)+\left(p 2 * \lambda_{2}\right)+\left(p 3 * \lambda_{3}\right)\right. \\
+ & \left.\left(p 4 * \lambda_{4}\right)\right]\left(4 \mu\left(1-p_{b}\right) \lambda_{b}+1\right)
\end{aligned}
$$

The four access categories have the independent arrival of traffic according to the type of traffic each access category is dealing with. But, there might be some sort of misdetection in choosing the access category by the incoming message. The probability of misdetection is represented by the symbol $p_{d}$. P1, p2, p3 and p4 are the representations for access probabilities of each access category respectively.

Collision Probabilities between access categories

$\zeta=p_{d}\left[\left(p 1 * n_{1}\right)+\left(p 2 * n_{2}\right)+\left(p 3 * n_{3}\right)+\left(p 4 * n_{4}\right)\right]$ $p_{d}$

$=$ probability of mis detection of the access category

$n 1$ is the number of vehicles in the access category, AC0 and expressed by the ratio of the back-off rate in AC0 to the transmitting rate. The expression is given below.

$$
n_{1}=\frac{\lambda_{1}}{\mu}
$$

$n 2$ is the number of vehicles in the access category, $\mathrm{AC} 1$ and expressed by the ratio of the back-off rate in $\mathrm{AC} 1$ to the transmitting rate. The expression is given below.

$$
n_{2}=\frac{\lambda_{2}}{\mu}
$$

$n 3$ is the number of vehicles in the access category, AC2 and expressed by the ratio of the back-off rate in AC2 to the transmitting rate. The expression is given below.

$$
n_{3}=\frac{\lambda_{3}}{\mu}
$$

$n 4$ is the number of vehicles in the access category, AC3 and expressed by the ratio of the back-off rate in AC3 to the transmitting rate. The expression is given below.

$$
n_{4}=\frac{\lambda_{4}}{\mu}
$$

\section{PERFORMANCE ANALYSIS}

In this section, the assessment of the throughput of IEEE 802.11p EDCA with drop limit analytically, in the assumption of ideal channel conditions (i.e. No hidden terminals) is given. In the analysis, it is considered that the frames are always available in the transmission queues of the vehicle. The analytical results related to the performance of the proposed model are given. The proposed model is used to analyse the performance of IEEE $802.11 \mathrm{p}$ MAC sub layer with the drop limit. The simulation parameters given in Table III are taken from the latest draft IEEE $802.11 \mathrm{p}$ standard.

Let $\tau$ is the transmission probability of a vehicle to transmit a frame in a particular slot. Any kind of transmission will be happened when the back-off counter is equal to zero, regardless of the retry limit and back-off stage $j$. The transmission probability can be expressed as

$$
\begin{aligned}
& \tau=\int_{j=0}^{m} \pi_{0} \\
& =\pi_{0}\left[\frac{1-p^{m}}{1-p}\right]
\end{aligned}
$$

Here $\mathrm{m}$ is the drop limit of the packet and $\mathrm{p}$ is the collision probability.

To determine the collision probability that a frame collides in a slot, the collision occurs only if at least one of the $(n-1)$ remaining vehicles transmits in the same time slot

$p=1-(1-\tau)^{n-1}$

After calculating $\tau$, the probability $p_{t r}$ is given that in a slot time there is at least one transmission, given active stations, and the probability $p_{s}$ that a transmission occurred is successful, can be expressed as

$p_{t r}=1-(1-\tau)^{n}$

$p_{s}=n \tau(1-\tau)^{n-1}$

Let $\mathrm{S}$ be the normalized saturated throughput. It is defined as the ratio of successfully transmitted payload bits to the time 
during the channel was used. Assume E[D] is the average data payload size. The throughput of each access category is given by as follows. The type of traffic ACO is having is "background". The throughput for access category AC0 is given by

$$
\text { Throughput }_{B K}=\frac{L}{T}\left(\pi_{0}+\pi_{1}+\pi_{b}\right)
$$

The type of traffic AC1 is having is "Best effort". The throughput for access category $\mathrm{AC} 1$ is given by

$$
\text { Throughput }_{B E}=\frac{L}{T}\left(\pi_{0}+\pi_{2}+\pi_{b}\right)
$$

The type of traffic AC2 is having is "Video". The throughput for access category AC2 is given by

$$
\text { Throughput }_{V I}=\frac{L}{T}\left(\pi_{0}+\pi_{3}+\pi_{b}\right)
$$

The type of traffic AC3 is having is "Voice". The throughput for access category AC 3 is given by

$$
\text { Throughput }_{V O}=\frac{L}{T}\left(\pi_{0}+\pi_{4}+\pi_{b}\right)
$$

Here, $R=$ Transmission rate,

$L=$ Packetsize transmitted by a vehicle, $T=$ Duration of the packet transmission and can be expressed as

$$
T=\frac{L}{R}
$$

\section{SIMULATION RESULTS}

In this section, the analytical results related to the performance of the proposed model are presented. The proposed model is used to analyse the performance of IEEE $802.11 \mathrm{p}$ in terms of throughput. The model is simulated using various performance metrics against throughput. The various
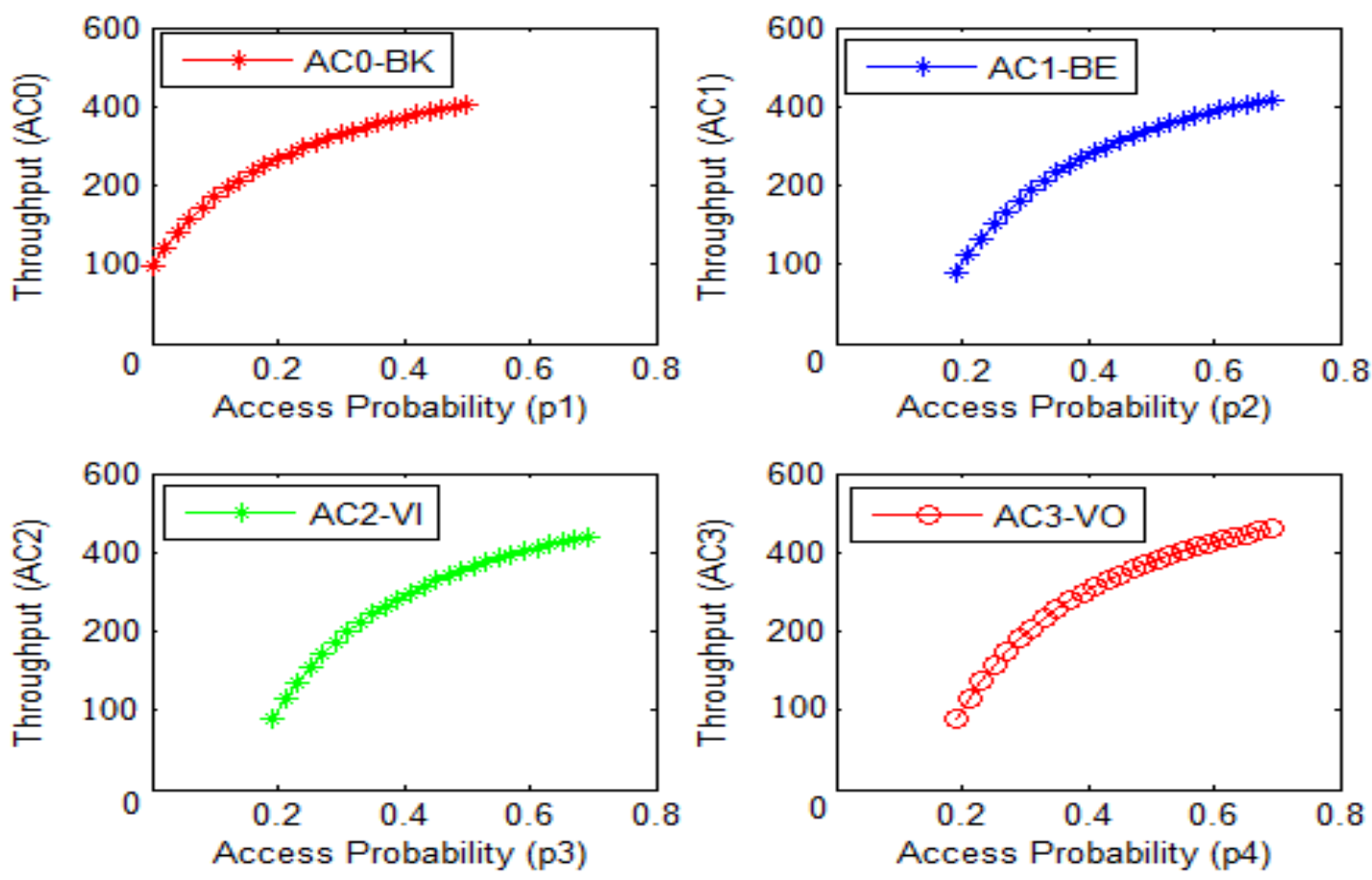

Figure 3: Throughput for varying access categories 
The transmission probability $(\tau)$ is dependent on collision probability and the size of contention window as well. The model is tested for varying number of nodes (vehicles). Figure 4 shows the effect of transmission probability for AC0 and $\mathrm{AC}$ over throughput. Initially, the throughput increases with transmission probability. After achieving the maximum value ( $\tau=0.05$ approx.), the throughput remains constant. For
$\mathrm{AC} 0$, the maximum throughput achieved is 1788 . For AC1 the maximum throughput achieved is 1787 because the density of nodes is low. Therefore, there will be less number of collisions. The throughput decreases with the increase in a number of nodes. Both the access categories have an almost same value of the throughput.
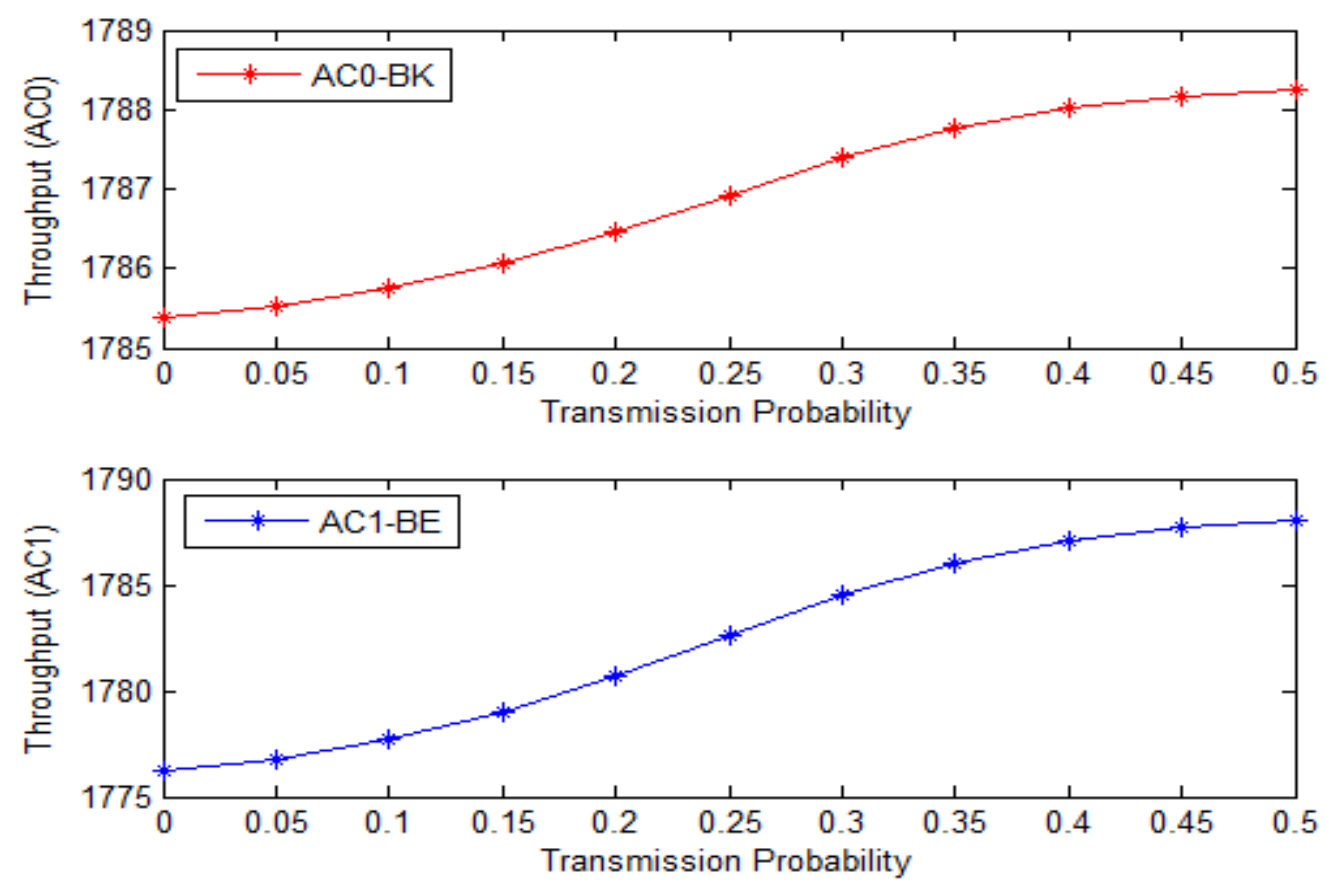

Figure 4: Throughput for varying transmission probability for AC0 and AC1
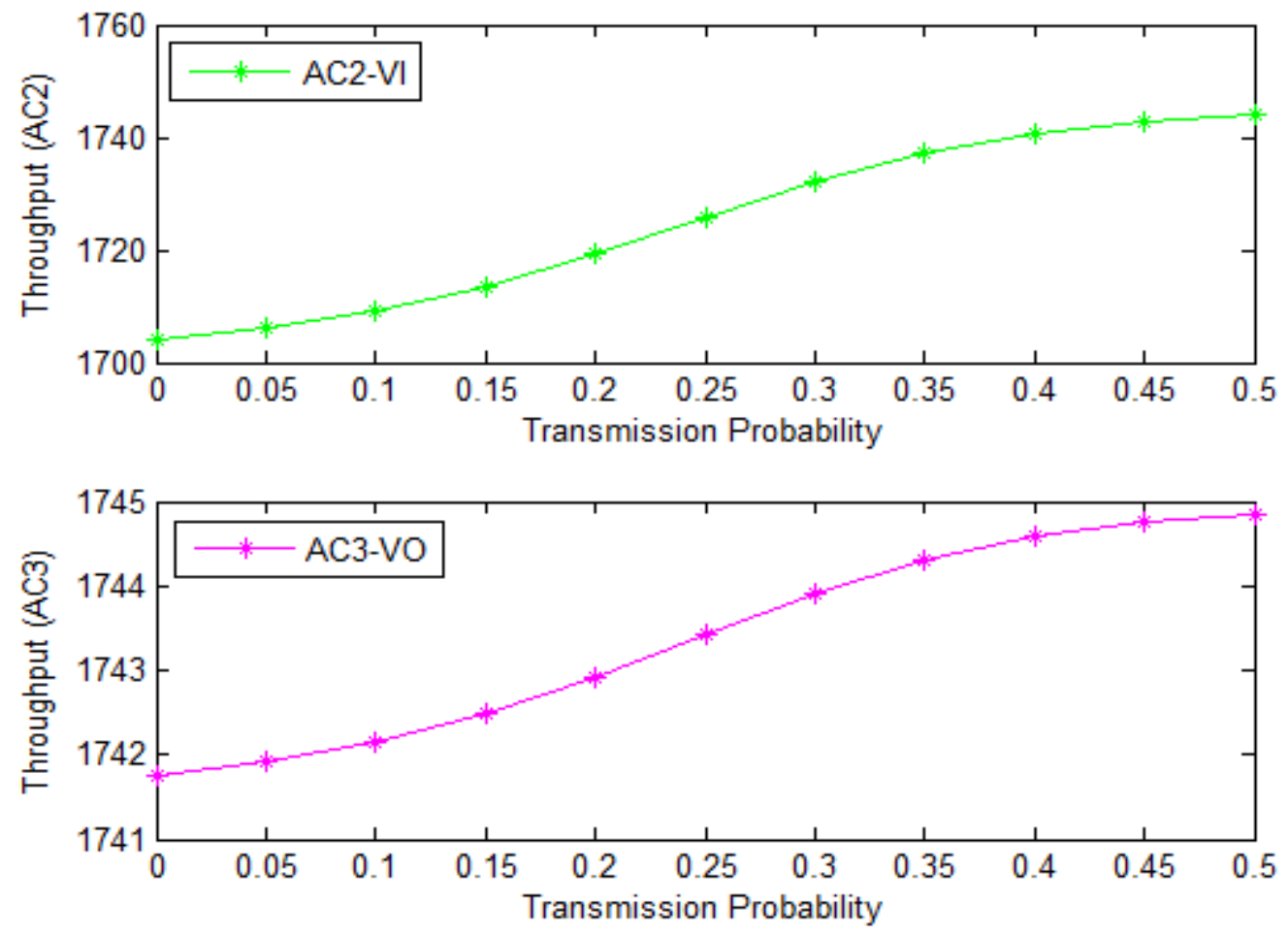

Figure 5: Throughput for varying transmission probability for AC2 and AC3 
As discussed before, EDCA categorizes the data on the basis of the type of application. So the highest priority data must be transmitted first than the lower priority data. The graph in figure 5 describes that transmission probability of voice traffic (AC3) is greater than the lower priority data category AC2. The figure has been drawn between the transmission probability on $\mathrm{x}$-axis and throughput on the $\mathrm{y}$-axis. The highest priority has been given to the access category 3 and the figure supports the same clearly. The throughput for AC3 is higher (1745) than AC2 (1740).

\section{CONCLUSION}

The paper evaluated the performance of an analytical model for IEEE $802.11 \mathrm{P}$ in terms of the throughput for four access categories separately. The comparison of all the access categories is also presented in the paper. The model considers the data packets and the control packets separately. The performance metric used for performance evaluation are throughput, transmission probability and the access probability. The proposed model had been created using the continuous time Markov chain (CTMC). The process of CTMC used in this paper is a birth-death process. The results show the effect of various performance metrics with respect to the throughput, transmission probability and access probability. The vehicular network is a emerging technology these days. There is a lot more future reseach scope. Some of them includes the improvement in the routing protocol for MAC layer in order to solve the hidden and exposed terminal problems in the vehicular adhoc ntworks.

\section{REFERENCES}

[1] G. Karagiannis, O. Altintas, E. Ekici, G. Heijenk, B. Jarupan, K. Lin and T. Weil, "vehicular networking: A survey and tutorial on requirements, architectures, challenges, standards and solutions,"IEEE communication surveys \& tutorials, vol. 13 , No. 4, pp. 584-616, 2011.

[2] Z. H. Mir and F. Filali, "LTE and IEEE 802.11p for vehicular networking: a performance evaluation", Eurasip journal on wireless communications and networking (Springer), vol. 2014, No. 1, pp. 89, 2014.

[3] R. Uzcategui and G. Acosta-Marum, "Wave: A tutorial," IEEE Communication Mag., vol. 47, no. 5, pp. 126-133, 2009.

[4] M. Torrent-Moreno, "Inter-vehicle communications: achieving safety in a distributed wireless environment," $\mathrm{PhD}$ dissertation, Inst. of Telematics, University Karlsruhe (TH), Karlsruhe, Germany, 2007.

[5] D. Jiang, L. Delgrosso, "IEEE 802.11p: Towards an international standard for wireless access in vehicular environments," IEEE Vehicular Technology Conference, VTC Spring, pp. 2036-2040, 2008.

[6] N. M. Rabadi, "Implicit certificates support in IEEE 1609 security services for wireless access in the vehicular environment (WAVE)," IEEE $7^{\text {th }}$ international conference on Mobile Adhoc and sensor systems (MASS), pp. 531-537, 2010.

[7] M. Amadeo, C. Campolo and A. Molinaro, "Enhancing IEEE802.11p/WAVE to provide infotainment applications," Elsevier Ad hoc Networks, vol. 10, issue 2, pp. 253-269, March 2012.
[8] T. H. Luan, X. Ling, X. Shen, "Provisioning QoS controlled media access in vehicular to infrastructure communications," Elsevier Ad hoc Networks, vol. 10, No. 2, pp. 231-242, 2012.

[9] Y. Xiao, "Performance analysis of priority schemes for IEEE 802.11 and IEEE 802.11e wireless LANs," IEEE transactions on wireless communications, vol. 4, No. 4, pp. 1506-1515, July 2005.

[10] Y. H. Bae, K. J. Kim, M. Moon, B. D. Choi, “Analysis of IEEE 802.11 non-saturated DCF by matrix analytical methods," Annals of Operations Research (Springer), vol. 162, No. 1, pp. 3-18, 2008.

[11] J. W. Robinson, and T. S. Randhawa, "Saturation Throughput Analysis of IEEE 802.11e Enhanced Distributed Coordination Function," IEEE journal on selected areas in communications, vol. 22, No. 5, pp. 917-928, June 2004

[12] E. Hamadani, M. Mostafavi, R. Tafazolli and V. Rakocevic, "Analysis of IEEE 802.11 DCF parameters on achievable throughput in ad hoc networks," Proceedings of IEEE $70^{\text {th }}$ vehicular technology fall conference (VTC 2009-Fall), pp. 1-6, 2009.

[13] R. Shirani, F. Hendessi, "A Markov chain model for evaluating the performance of the store-carry-forward procedure in VANETs" Proceedings of IEEE $11^{\text {th }}$ Singapore international conference on communication systems (ICCS 2008), pp. 593-598, 2008.

[14] L.A. Pipes, "An operational analysis of traffic dynamics," Journal of Applied Physics, AIP, vol. 24, no. 3, pp. 274-281, March 1953.

[15] K.Nagel, M. Schreckenberg, "A cellular automaton model for freeway traffic," Journal de physics I France, EDP Sciences, vol. 2, no. 12, pp. 2221-2229, Dec. 1992.

[16] B. Nithya, C. Mala and V. Kumar, "Simulation and performance analysis of various IEEE 802.11 backoff algorithms," $2^{\text {nd }}$ International conference on communication, computing and security [ICCCS-2012], Elsevier, vol. 6, pp. 840-847, 2012.

[17] F. Cali, M. Conti, E. Gregori, Dynamic tuning of the IEEE 802.11 protocol to achieve a theoretical throughput limit, IEEE/ACM Transaction on Networking, vol. 8, No. 6, pp. 785-799, 2000.

[18] Khabazian, M., Mehmet-Ali, M., \& Aissa, S. (2013) Analysis of continuous communication availability in vehicular ad hoc networks. IEEE Systems Journal, 7(1), 137-150.

[19] Abboud, K., \& Zhuang, W. (2016). Stochastic modelling of single-hop cluster stability in vehicular ad hoc networks. IEEE Transactions on Vehicular Technology, 65(1), 226-240.

[20] Mokdad, L., Ben-Othman, J., \& Ballarini, P. (2016, June). Stochastic models for IEEE $802.11 \mathrm{p}$ In Computers and Communication (ISCC), 2016 IEEE Symposium on (pp. 74-78). IEEE.

[21] Giang, A. T., Busson, A., \& Di Renzo, M. (2016) Modelling and optimization of CSMA/CA in VANET. Annals of Operations Research, 239(2), 553568. 